\title{
Signaling and Scheduling WIMAX Gateway Design Based on Resource Reservation Protocol
}

\author{
Mahmoud Ahmad Albawaleez*, Kamaruzzaman Seman, \\ Kamarudin Saadan and Khairi Abdulrahim \\ Universiti Sains Islam Malaysia (USIM), Bandar Baru Nilai, 71800 Nilai, Negeri Sembilan, Malaysia; \\ mahmoud_bawaleez@yahoo.com,drkzaman@usim.edu.my, kamarudin@usim.edu.my, khairiabdulrahim@usim.edu.my
}

\begin{abstract}
Objectives: As a result of signal transmission in WiMAX too long distance, and because there are many request packets arrive at WiMAX gateway request handovers, this cost a congestion which may result in loss or delay in packet time sensitive. The objectives of this paper are to investigate the delay when packets with real time sensitive handover in WiMAX gateway and to measure and enhance the handover performance. Methods/Statistical Analysis: To overcome this problem, and achieve the objectives is propose a new scheme Resource Reservation Protocol \& Random Early Detection with Weighted Fair Queuing (RR-WFQ) in order to analyze and enhance delay for real time packet in WiMAX gateway. This schema applies all Resource Reservation Protocol (RSVP), Random Early Detection (RED), and Weighted Fair Queuing (WFQ) as a Scheduling, RED as a mechanism and RSVP as a signaling protocol. Findings: The proposed scheme enhances the QoS by minimizing the delay in WiMAX gateway and BS. The scheme enhances the delay in all queues in a gateway, and video conferencing. The result of the scheme shows an enhancement delay in the WiMAX gateway. Application/ Improvements: Mathematical analysis and modeling have been done using queuing theory. Simulation has been done using OPNET simulation. Simulation results for the new approach confirms that has minimized delay in WiMAX gateway and BS the gateway and enhances the QoS.
\end{abstract}

Keywords: Signaling Protocol, Proposed RR-WFQ Scheme, Queuing Management, Resource Reservation Protocol, WIMAX Scheduling

\section{Introduction}

In WiMAX communication area good coverage, low cost, and better data rate are the goals of designers. Signaling real time packet sensitive in WiMAX is an open issue because it is unspecified by IEEE 802.16-2004, IEEE $802.16 \mathrm{~d}$, IEEE $802.16 \mathrm{e}$ or IEEE $802.16 \mathrm{j}^{1}$. Congestion always happens in WIMAX gateway because of the huge number of packets arrive at the gateway and requesting handover. Since no network can guarantee hundred percent the QoS, which result to packet lost and delay². A protocol like RSVP is provided in order to enhance QoS End-to-End delay, loss, delay variation and throughput ${ }^{3}$. The real goals of RSVP are to improve the heterogeneous network, to be flexible with the direction of transmission, and to make use of all the resources ${ }^{4}$. While Random
Early Detection (RED) is considered as a queue mechanism to avoid congestion, modified RED has been applied with WFQ to avoid congestion, and shows good result in minimizing the delay in WIMAX BS and WIMAX gateway, this scheme called Nilai scheme ${ }^{5}$.

\section{Queuing Theory}

$\mathrm{M} / \mathrm{M} / 1$ is a queuing theory with possion arrive process for packets, exponentially service for those packets, and a single server with an infinite length of queue, $\lambda$ is the arrival rate, and $\mu$ is the service rate with average services equally to $1 / \mu^{6}$; Figure 1 shows the M/M/1 structure, where all arrival rate, service rate, and average services explained.

*Author for correspondence 


\section{Arrival process wile $\lambda$ arrival rate}

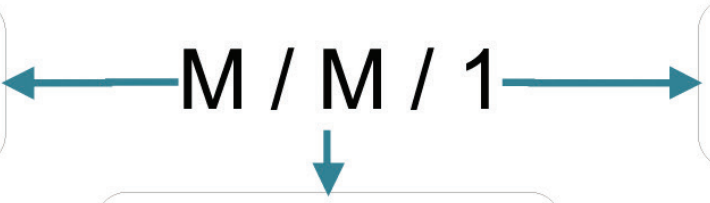

Single servers with average services $1 / \mu$

\section{Service Process \\ wile $\mu$ service rate}

Figure 1. $\mathrm{M} / \mathrm{M} / 1$ structure.

Property $\rho$ for $\mathrm{M} / \mathrm{M} / 1$ is the arrival rate over service rate which is equal to $\lambda / \mu$ where $\lambda / \mu<1$, so if arrival rate is more than service rate $(\lambda>\mu)$ it will be at least one packet and will never be served or worst this packet will drop ${ }^{7}$.

Because Markov Chain has memory less property, "memoryless property a random variable $\bar{x}$ is said to be memoryless if, and only if for every $\alpha, \beta \geq 0$ "

$\mathrm{M} / \mathrm{M} / 1$ follow time dependent which is long term behaved under stochastic equilibrium?

$\lim _{t \rightarrow \infty} \rho_{i j}(s, t)=\rho_{j} \rightarrow s<t$

According to ${ }^{10}$ in $\mathrm{M} / \mathrm{M} / 1$ queuing system is the output and input for each state follows stochastic equilibrium which is also known as global balance; Figure 2 shows the Global balance based on $\mathrm{M} / \mathrm{M} / 1$.

$\bar{N}$ is the number of packet in the system wither they are waiting in queue or being serviced to be written as Formula (2).

$$
\begin{aligned}
& \bar{N}=\sum_{j=0}^{\infty} j \rho_{j}=\sum_{j=0}^{\infty} j(1-\rho) \rho^{j}=(1-\rho) \sum_{j=0}^{\infty} j \rho^{j} \\
& =(1-\rho) \frac{\rho}{(1-\rho)^{2}}=\frac{\rho}{(1-\rho)}
\end{aligned}
$$

In order to calculate delay on WiMAX based on $\mathrm{M} / \mathrm{M} / 1$ we use Formula (3).

$\bar{D}_{M / M / 1}=\sum_{j=0}^{\infty} \bar{D}_{j} \cdot \rho_{j}=\sum_{j=0}^{\infty}\left(\frac{j+1}{\mu}\right) \rho_{j}=\frac{\bar{N}+1}{\mu}=\frac{\frac{\rho}{1-\rho}+1}{\mu}=\left(\frac{1}{\mu}\right)\left(\frac{1}{1-\rho}\right)=\left(\frac{1}{\mu}\right)\left(\frac{1}{1-\frac{\lambda}{\mu}}\right)=\frac{1}{\mu-\lambda}$

Figure 3 shows that in WiMAX handover from BS to another BS process flows the global balance which is in another word M/M/1 system. Formula (4) are used to calculate the total delay in all handover process.

$$
\bar{D}_{M / M / 1}=\bar{D}_{\text {processing }}+\bar{D}_{\text {Time spend in the queue }}
$$

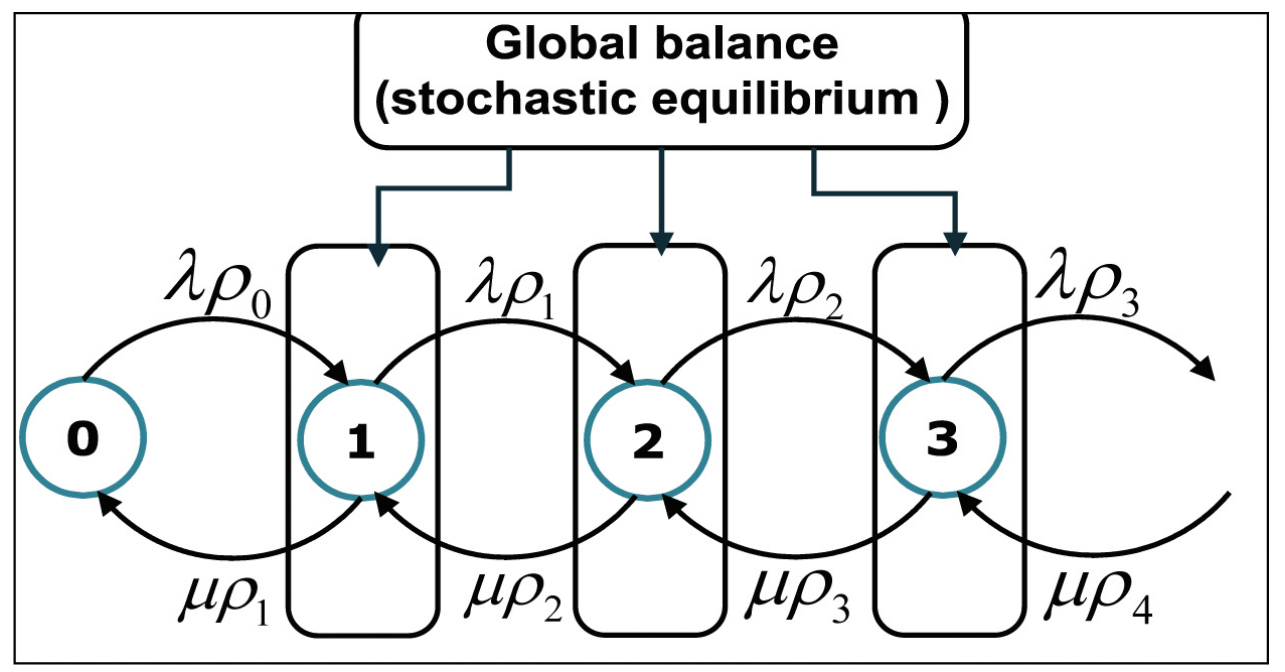

Figure 2. Global balance. 


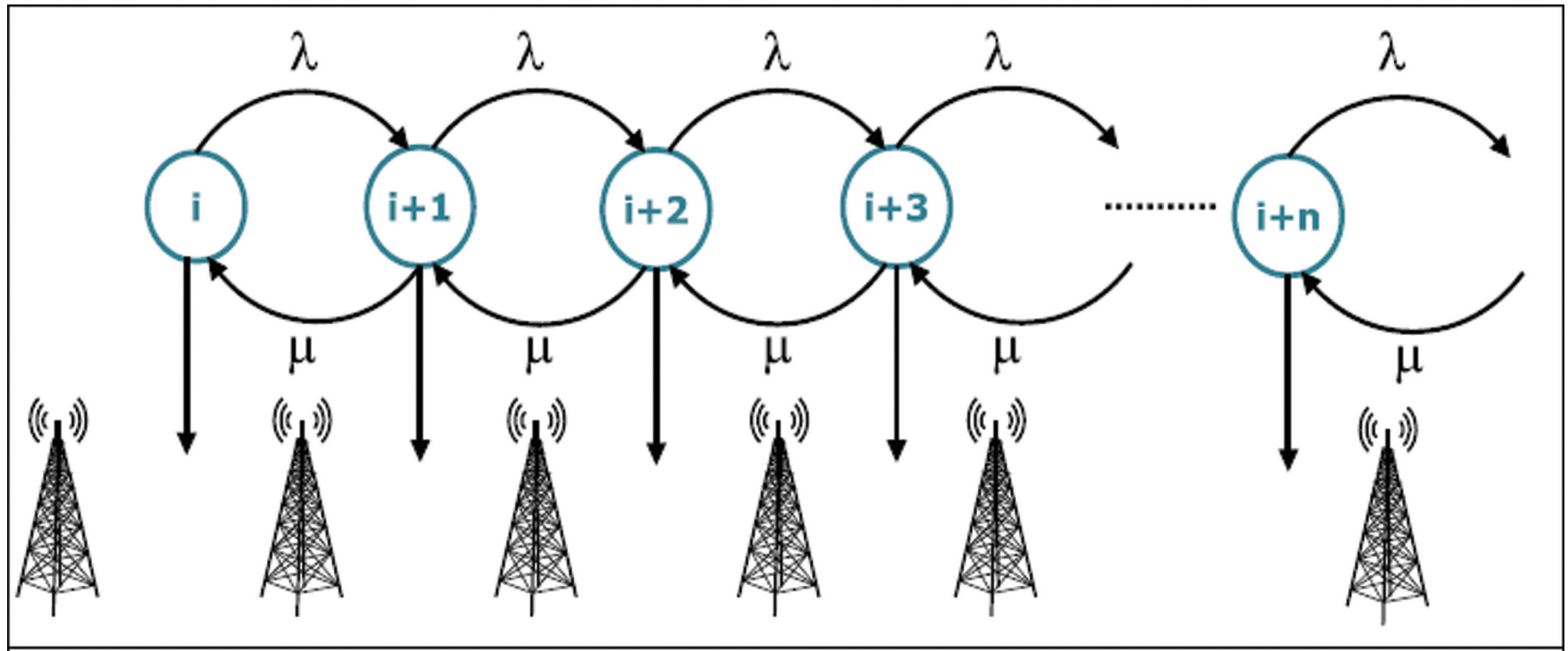

Figure 3. $\mathrm{M} / \mathrm{M} / 1$ on WiMAX.

\section{WFQ (Weighted Fair Queuing)}

WFQ is scheduling mechanism deployed for packets in network, it is used in all getaways, switches and routers to provide QoS and guarantee less delay and less loss to packets, the virtual starting time in the gateway based on WFQ will be defined as Formula(5)플.

$$
S_{i}^{j}=\max \left\{S_{i}^{j-1}, V(t)\right\}+\frac{L_{i}^{j}}{\phi_{i}}
$$

$S_{i}^{j}$ : The virtual starting time.

$V(t)$ : The starting time for packet to arrive.

$L_{i}^{j}$ : Size of packet.

$\phi_{i}:$ The distribution coefficient

$\left\{S_{i}^{j-1}, V(t)\right\}:$ The virtual starting for gateway.

Where delay in WFQ is defined as Formula (6).

$$
\bar{D}_{W F Q}=\frac{\max _{t h}}{\rho}+\frac{L_{\max }}{C}
$$

Scheme such Weighted Random Early Detection (WRED) Scheduler have been presented by $\underline{12}$ in order to avoid congestion for real time packet sensitive.

\section{Resource Reservation Protocol (RSVP)}

RSVP is a signaling protocol located on transport layer aim to clear path to packet required QoS $\underline{13}$. Protocol such (RSVP, RSVP-TE, and CR-LDP) are very important when it comes for real time packet sensitive ${ }^{\underline{14}}$, RSVP a protocol has provided as a solution for transmission delay especially for packet time sensitive ${ }^{\underline{15}}$. The reason of using RSVP is that it can work with any communication network includes CAC (Connection Admission Control), scheduler, control policy, and classifier because resources in that communication should be according to QoS $\underline{16}$.

\section{The Proposed Scheme}

The scheme (RR-WFQ) proposed to modify for Nilai scheme, where extend queue have been added in WiMAX gateway and BS. Different mechanisms in order to enhance and improve WiMAX gateway and BS have been applied. to calculate the maximum delay in WiMAX gateway when all RSVP signaling protocol, RED as a detection mechanism, and WFQ as a scheduling are applied. And compare with other techniques. Since Random Early Detection (RED) is calculated in in Formula (7), and the maximum delay is calculated as Formula (8). 


$$
\begin{aligned}
& \rho_{b}=\rho_{\max } \frac{C-\min _{t h}}{\max _{t h}-\min _{t h}} \\
& \bar{D}_{\text {max }}=\bar{D}_{W F Q}+\bar{D}_{M / M / 1}
\end{aligned}
$$

By applying all formulas 3,6, and 7 we will come to new formula to calculate total maximum delay $\bar{D}_{\max }$ as Formulas 9.

$$
\bar{D}_{\max }=\frac{\max _{t h}}{\rho_{\max } \frac{C-\min _{t h}}{\max _{t h}-\min _{t h}}}+\frac{L_{\max }}{C}+\frac{1}{\mu-\lambda}
$$

Figure 4 explains the modifying pseudo code for the proposed scheme, as well as Figures 5 showing that the modification has been done for Nilai scheme in both BS and WiMAX gateway to achieving the proposed scheme. The parameters used in the proposed RR-WFQ scheme are shown in Table 1.
Table 1. Proposed RR-WFQ scheme parameters

\begin{tabular}{ll}
\hline Parameters & Parameters Values \\
\hline$p_{\max }$ & Maximum marking probability \\
$p_{b}$ & Drooping probability \\
$\max _{t h}$ & Maximum threshold \\
$\min _{t h}$ & Minimum threshold \\
$C$ & Average queue length \\
$\bar{N}$ & Number of packets inside the queue \\
$\lambda$ & Packet arrive \\
$\bar{D}_{\max }$ & Total Maximum Delay \\
$\bar{D}_{W F Q}$ & Maximum Delay in WFQ \\
$\bar{D}_{M / M / 1}$ & Maximum Delay in M/M/1 Queuing \\
$L_{\max }$ & Mystem \\
\hline
\end{tabular}

\section{Applied RSVP \\ Applied RED \\ \#set $\lambda$ as packet arrive \\ \#set $\mathrm{C}$ as Average queue length \\ IF Max Threshold $<\mathrm{C}$ then Extend Packet \\ \{ \\ If ( Probability Packet for Dead line >=1) drop \\ Else \\ If ( Probability Packet for Dead line $<1$ ) Serve \\ \} \\ IF Min Threshold $<=\mathrm{C}$ or $\mathrm{C}<=$ Max Threshold \\ Then Calculate $\lambda$ Dropping Probability \\ IF Dropping Probability High Drop Packet \\ Else Enqueuse \\ IF C $<$ Min Threshold then Enqueue}

Figure 4. RR-WFQ pseudo code. 


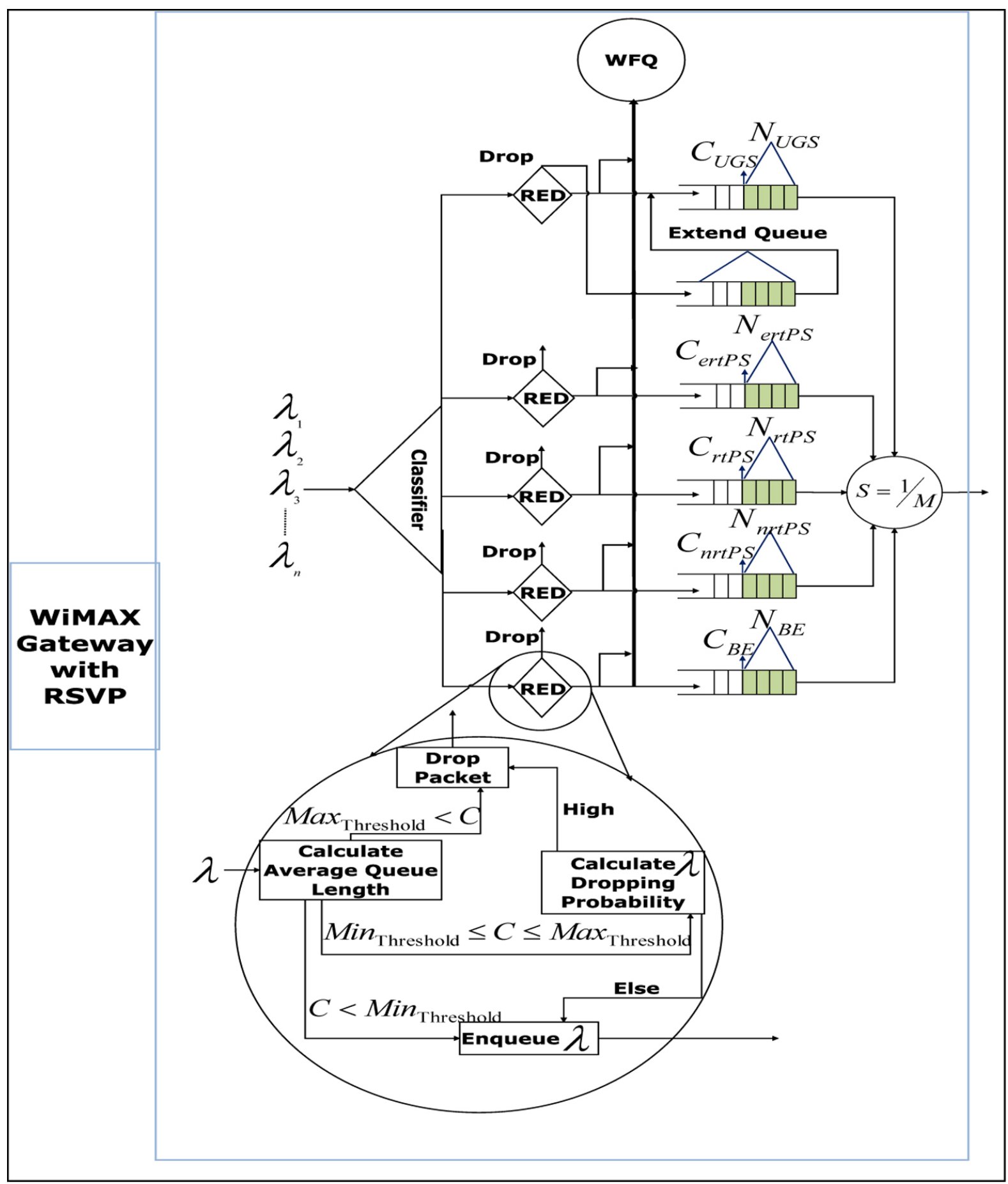

Figure 5. The proposed RR-WFQ scheme.

\section{Simulation Model and Results}

The simulation model shown in Figure 6, is proposing that scheme (RR WFQ) has been simulated and implemented using OPNET ${ }^{17}$. MAC and PHY layer parameter are exhibited in Table 2. Simulation was conducted for three scenarios: scenario one where FIFO applied as scheduling mechanisms, scenario two where MDRR applied as scheduling mechanisms, and the third scenario was the proposed scheme. Results of figures 7-9 show that the proposed scheme enhances the delay in WiMAX gateway. 
Table 2. Simulation parameters

\begin{tabular}{|ll|}
\hline \multicolumn{1}{|c|}{ Parameters Type } & \multicolumn{1}{c|}{ Parameters Values } \\
\hline Maximum SS & 100 \\
Maximum Distance & $6 \mathrm{~km}$ \\
Transport protocol type & $\mathrm{UDP} / \mathrm{TCP}$ \\
Symbol Duration & $102.86(\mathrm{n}=28 / 25$, delta_f $=10.94 \mathrm{kHz}, \mathrm{Tg}=\mathrm{Tb} / 8)$ \\
Base Frequency & $5 \mathrm{GHz}$ \\
PHY Profile & Wireless OFDMA $20 \mathrm{MHz}$ \\
Bandwidth & $20 \mathrm{MHz}$ \\
Frame Duration & $5 \mathrm{~ms}$ \\
DL/UL Zone & $100 \%$ \\
DL/UL Usage Mode & PUSU \\
Duplexing Technique & TDD \\
PHY & OFDMA \\
Fast Fourier Transform Size & 1024 \\
\hline
\end{tabular}

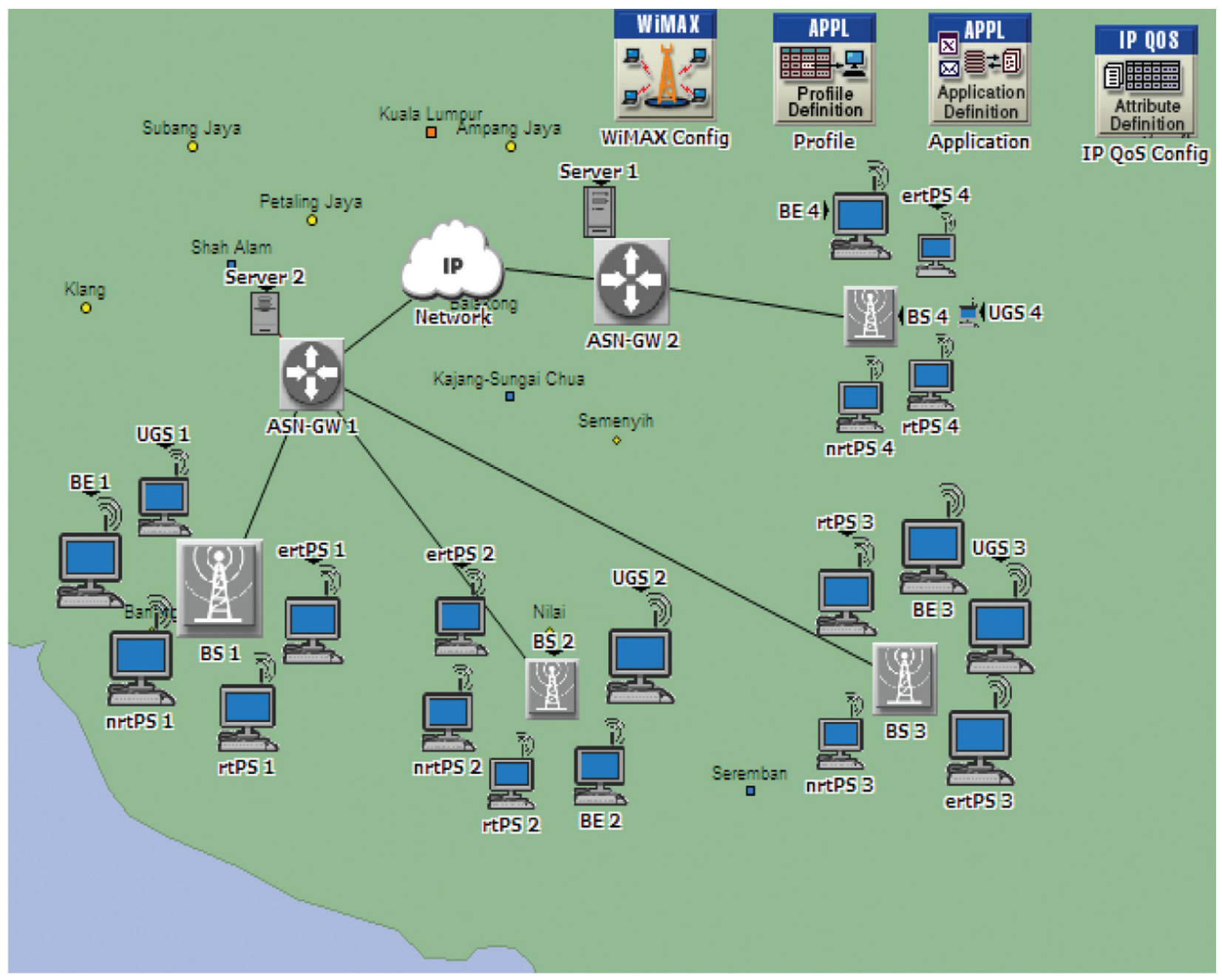

Figure 6. OPNET project model for RR-WFQ scheme. 


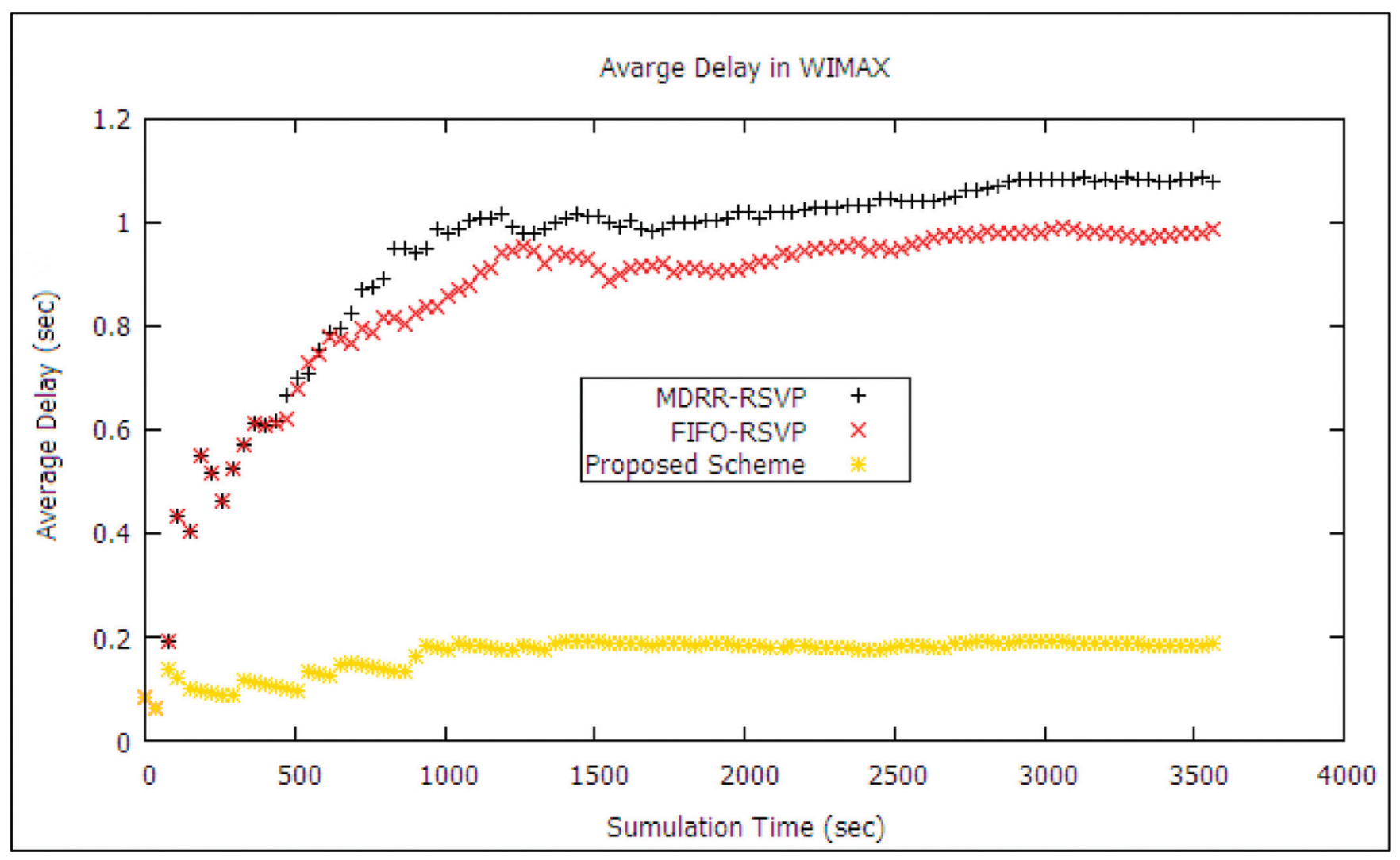

Figure 7. Average delay in WIMAX.

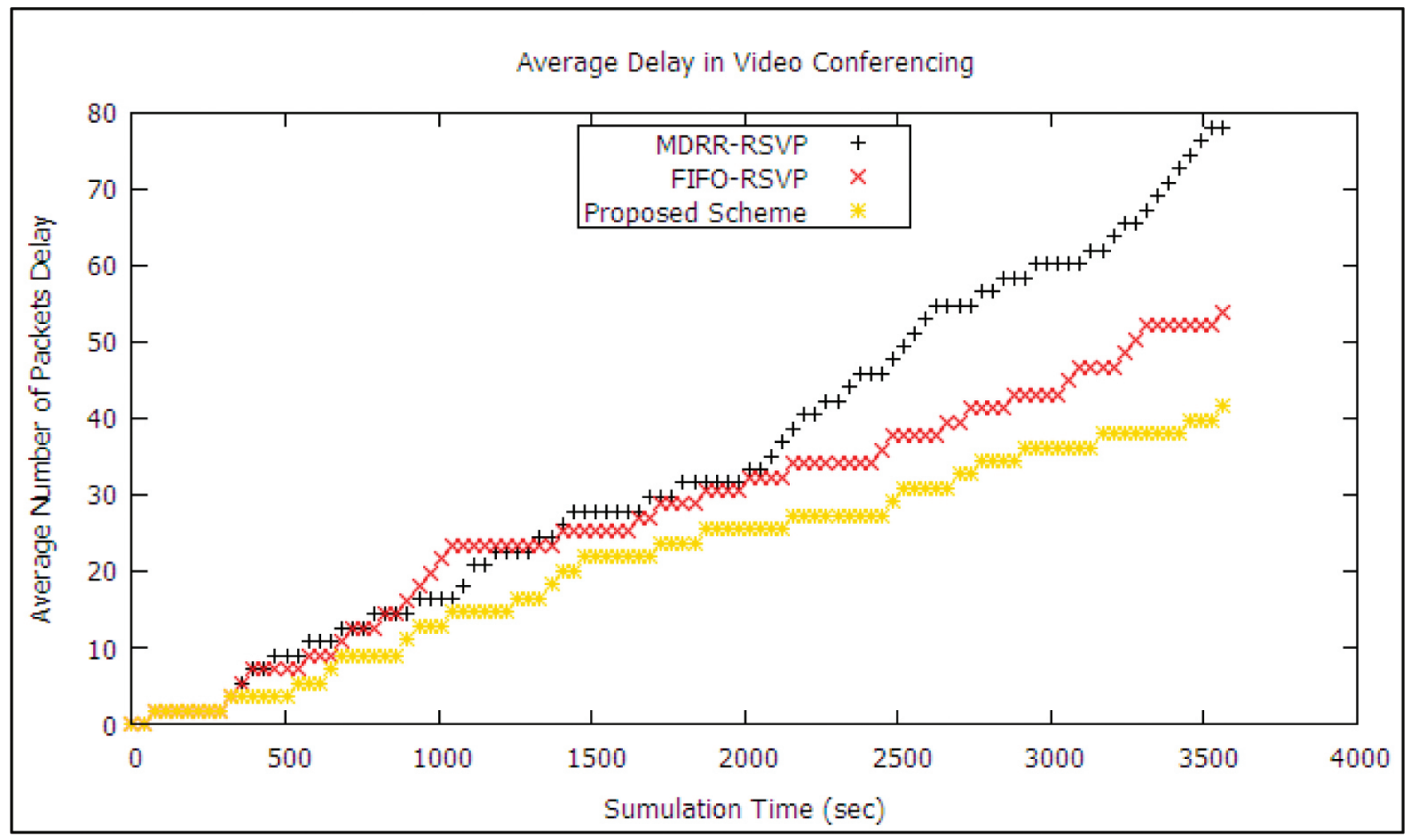

Figure 8. Average delay in video conferencing. 
Figure 7 demonstrates the average in WiMAX gateway. The proposed RR-WFQ scheme reduces delay and enhances the QoS for real time packet sensitive compare to other scheme like MDRR-RSVP, and FIFO-RSVP.

Figure 8 demonstrates the average delay for a number of packets in video conferencing in WiMAX gateway. The proposed RR-WFQ scheme reduces delay and enhances the QoS for real time packet sensitive compared to other schemes like MDRR-RSVP, and FIFO-RSVP.

Figure 9 demonstrates the average delay packets for queue in WiMAX gateway. The proposed RR-WFQ scheme reduces delay and enhances the QoS for real time packet sensitive compare to other scheme like MDRRRSVP, and FIFO-RSVP.

Results of the proposed scheme have been compared with other schemes. The proposed scheme enhances the performance of WiMAX gateway. The qualitative results have shown Figure 9 enhancement in delay. Table 3 shows the quantitative results.
Table 3. Quantitative results for proposed scheme

\begin{tabular}{|c|c|c|c|}
\hline \multicolumn{4}{|c|}{ Average Delay (sec) } \\
\hline & MDRR-RSVP & FIFO-RSVP & RR-WFQ \\
\hline $\begin{array}{l}\text { WIMAX } \\
\text { Gateway }\end{array}$ & $0.835893 \mathrm{sec}$ & $0.772738 \mathrm{sec}$ & $0.158419 \mathrm{sec}$ \\
\hline $\begin{array}{l}\text { Queue in } \\
\text { Gateway }\end{array}$ & $0.016979 \mathrm{sec}$ & $0.018111 \mathrm{sec}$ & $0.012728 \mathrm{sec}$ \\
\hline \multicolumn{4}{|c|}{ Average Delay Packet Number } \\
\hline & MDRR-RSVP & $\begin{array}{l}\text { FIFO- } \\
\text { RSVP }\end{array}$ & RR-WFQ \\
\hline $\begin{array}{l}\text { Video } \\
\text { Conferencing }\end{array}$ & 30.11479 & 25.33266 & 19.85494 \\
\hline
\end{tabular}

\section{Conclusion}

Packets with real time sensitive are very important in the communication area. Using RSVP signaling protocol, the RED detection mechanism, and WFQ scheduler is better than other schemes like FIFO and MDRR for real time packet sensitive. A modifying for Nilai scheme has been

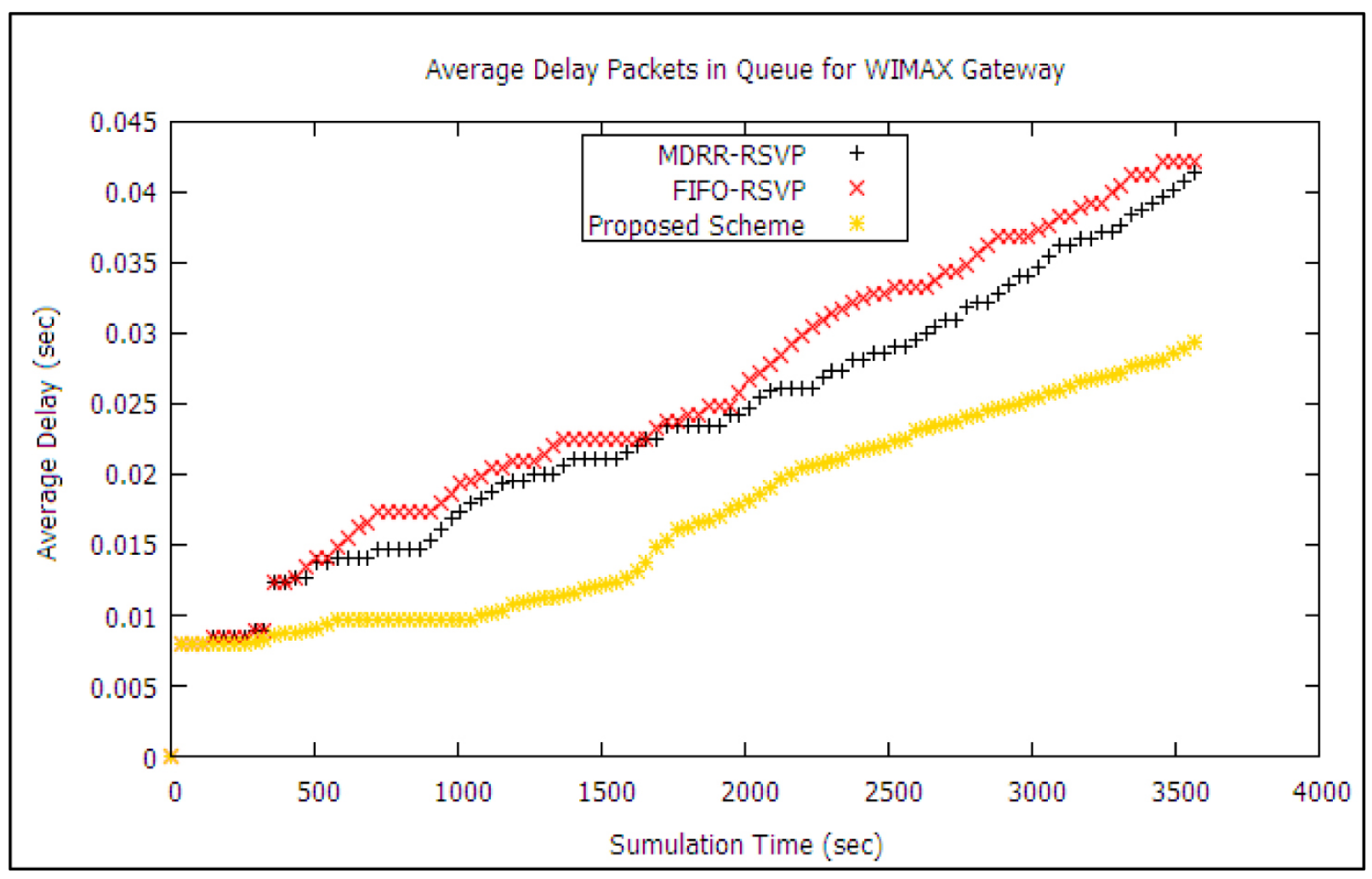

Figure 9. Average delay packets for queue in gateway. 
proposed. The proposed scheme has enhance the QoS by minimizing the delay in WiMAX gateway and BS. The proposed scheme has considered $\mathrm{M} / \mathrm{M} / 1$ queuing theory as a mathematical model in order to control the number of packet process in the WiMAX gateway. The scheme enhances the delay in all queues in a gateway, and video conferencing. For future work, the proposed scheme could be tested for PCS or non real time packet sensitive.

\section{References}

1. Albawaleez MA, Seman K, Saadan K, Abdulrahim K, Eds. Priority Control Scheduling for Downlink and Uplink in WIMAX Network. The Second International Conference, Technological Advances in Electrical, Electronics and Computer Engineering (TAEECE); 2014. p. 1-5.

2. Huang J, Huang CT. Design and verification of secure mutual authentication protocols for mobile multi hop relay WIMAX networks against rogue base/relay stations, Journal of Electrical and Computer Engineering. 2016; 1-12. Crossref. Crossref.

3. Fathima KM. A content-centric efficient search with structured overlays in $\mathrm{P} 2 \mathrm{P}-\mathrm{VoD}$ system. International Conference, Intelligent Systems and Control (ISCO); 2016. p. 1-6.

4. Thulasiraman P, Sagir Y. Dynamic bandwidth provisioning using Markova chain based RSVP for unmanned ground networks. IEEE International Inter-Disciplinary Conference, Cognitive Methods in Situation Awareness and Decision Support (COGSIMA); 2014. p. 130-36. Crossref.

5. Albawaleez MA, Seman K, Saadan K, Abdulrahim K. Signaling and scheduling WIMAX gateway design based on early congestion detection, Asian Academic Research Journal. 2017; 4(11):223-33.

6. Mieghem PV. Performance analysis of communications networks and systems. Cambridge: Cambridge University Press; 2009.
7. Ruggeri F, Wiper MM, Ríos Insua D. Bayesian analysis of stochastic process models, Wiley. 2012; 1-332.

8. Daigle JN. Queueing theory with applications to packet telecommunication, Springer Science and Business Media. 2005; 1-342. Crossref, Crossref.

9. Bhat UN. An introduction to queueing theory: Modeling and analysis in applications, Birkhäuser. 2015; 1-14. Crossref, Crossref. PMCid:PMC4424041.

10. Ng CH, Boon-Hee S. Queueing modelling fundamentals: With applications in communication networks. John Wiley \& Sons, 2008. Crossref.

11. Mitrou N. Networking 2004: Networking technologies, services, and protocols; Performance of computer and communications networks; Mobile and wireless communications. Third International IFIP-TC6 Networking Conference, Athens, Greece, Proceedings: Springer Science and Business Media; 2004 May. 3042, p. 1-1520.

12. Alam M, Latif S, Akter M, Arafat M, Hakak S. Performance analysis of MAC layer scheduling schemes for IMM applications over high speed wireless campus network in IEEE802, 11e. Indian Journal of Science and Technology. 2015; 8(3):53-61. Crossref.

13. Villapol ME, Billington J. Modelling and initial analysis of the resource reservation protocol using coloured petri nets. Proceedings of the Workshop on Practical Use of HighLevel Petri Nets, Aarhus, Denmark, 2000. p. 1-362.

14. Bensalah F, Kamoun NE, Bahnasse A. Scalability evaluation of VOIP over various MPLS tunneling under OPNET modeler, Indian Journal of Science and Technology. 2017; 10(29):1-8. Crossref.

15. Lin Z, Yingmei C, Zhen L, Zhuzhen H. An improved video monitoring system based on RSVP protocol. International Conference, Intelligent Transportation, Big Data and Smart City (ICITBS); 2015. p. 94-97. Crossref.

16. Durham D, Yavatkar R. Inside the Internet's resource reservation protocol: Foundations for quality of service. John Wiley \& Sons, Indian Nursing Council, 1999.

17. Sethi AS, Hnatyshin VY. The practical OPNET user guide for computer network simulation. CRC Cyclic redundancy checks Press; 2012. p. 1-527. 